\title{
Evaluación del consumo de alcohol en adolescentes mexicanos: un enfoque desde la economía conductual
}

\author{
Sarahí Alanís Navarro', Shoshana Berenzon Gorn², Francisco Lorenzo Juárez García ${ }^{2}$ \\ I Programa de Doctorado en Psicología, Facultad de Psicología, Universidad Nacional Autónoma de México \\ 2 Instituto Nacional de Psiquiatría Ramón de la Fuente Muñiz
}

\section{RESUMEN}

Introducción: el impacto de los programas para prevenir el consumo de alcohol en adolescentes puede incrementarse al incorporar un componente ambiental. En este trabajo se explora la utilidad de la economía conductual para analizar la preferencia por la sustancia, en función de las variables ambientales. Objetivo: evaluar el nivel de ingesta de alcohol en un grupo de adolescentes mexicanos, según el sexo, la edad, el acceso al consumo y el reforzamiento derivado de las actividades libres de sustancias. Método: participaron 7,569 estudiantes de bachillerato: $51 \%$ hombres y $49 \%$ mujeres. Se utilizó un modelo de regresión lineal múltiple para evaluar de qué manera las variables seleccionadas se relacionan con el nivel de consumo de alcohol. Resultados: el modelo de regresión se ajustó $(F=202.423, p<0.01)$ y explicó $33.6 \%$ de la varianza $\left(R^{2}=0.336\right)$. El sexo (ser hombre), la edad y el acceso al alcohol en contextos sociales y familiares se asociaron de manera positiva con el nivel de consumo. Las actividades académicas, las prosociales y las culturales se relacionaron negativamente con la conducta de consumo, mientras que el uso de medios electrónicos, las actividades sociales, las físicas y las recreativas tuvieron una relación positiva. Discusión y conclusiones: es necesario reducir el acceso al consumo de alcohol, tanto en el ámbito comercial como en los contextos sociales y familiares. Las intervenciones preventivas deben promover el involucramiento de los adolescentes en las actividades incompatibles con el consumo e incorporar un componente social para incrementar su nivel de reforzamiento.

Palabras clave: prevención, adolescentes mexicanos, economía conductual, acceso al consumo de alcohol, actividades libres de sustancias.

\begin{abstract}
Introduction: the impact of programs to prevent alcohol consumption in adolescents can be increased by incorporating an environmental component. This paper explores the usefulness of behavioral economics to analyze the preference for the substance as a function of environmental variables. Objective: to evaluate the level of alcohol consumption in a group of Mexican adolescents, as a function of sex, age, access to consumption and reinforcement derived from substance-free activities. Method: a total of 7,569 high school students participated: $51 \%$ were male and $49 \%$ female. A multiple linear regression model was used to evaluate how the selected variables are related to the level of alcohol consumption. Results: the regression model was adjusted ( $F=$ 202.423, $p<0.01)$ and explained $33.6 \%$ of the variance $\left(R^{2}=0.336\right)$. Gender (being male), age, and access to consumption in social contexts were positively associated with the level of alcohol consumption. Academic, prosocial and cultural activities were negatively related to alcohol consumption; while the use of electronic media, social, physical and recreational activities had a positive relationship. Discussion and conclusions: tit is necessary to reduce access to alcohol consumption, both commercially, and in social and family contexts. Preventive interventions should promote the involvement of adolescents in activities incompatible with consumption and incorporate a social component to increase their level of reinforcement.
\end{abstract}

Keywords: prevention, Mexican adolescents, behavioral economics, access to alcohol consumption, substance-free activities.

\footnotetext{
Autor de correspondencia:

Sarahí Alanís Navarro. Teléfono: 777327293

Correo electrónico: alaniszarah@gmail.com

Recibido: 06 de octubre de 2020

Aceptado: 26 de febrero de 2021

doi: 10.28931/riiad.2021.1.02
} 


\section{INTRODUCCIÓN}

Entre los adolescentes mexicanos, tanto las primeras causas de muerte (accidentes automovilísticos, suicidios y homicidios) como los principales problemas que requieren el uso de servicios de urgencias (violencia, traumatismos y fracturas) y de salud mental (depresión, ideación e intento suicida) se relacionan con el uso de sustancias, en particular con el alcohol (Borges et al., 2008; Borges et al., 2010; Guerrero et al., 2013). Según la Encuesta Nacional de Consumo de Drogas, Alcohol y Tabaco (ENCODAT), en el último año $15.2 \%$ de los adolescentes consumió alcohol de manera excesiva (Villatoro et al., 2017). Entre la población estudiantil, este patrón de consumo fue tres veces mayor en los estudiantes de bachillerato, en comparación con los de secundaria: $22.7 \%$ vs. $8.6 \%$ (Villatoro et al., 2015).

Los resultados de diversas revisiones sistemáticas y meta-análisis coinciden en señalar que los programas preventivos que integran el entrenamiento en habilidades figuran entre los más efectivos; sin embargo, también se ha observado que su efecto es pequeño y permanece por un corto periodo (Babor et al., 2010; Cuijpers, 2003; Foxcroft \& Tsertsvadze, 2011; Stockings et al., 2016). Las estrategias que han mostrado ser más efectivas son aquellas que incorporan restricciones ambientales dirigidas a incrementar el costo del alcohol, mediante el aumento en los impuestos, las prohibiciones del consumo en espacios públicos, la restricción de los horarios de venta, entre otras (Babor et al., 2010; Medina-Mora et al., 2010; Stockings et al., 2016). En conjunto, los resultados sugieren que el impacto de los programas preventivos basados en variables individuales puede incrementarse al incorporar un componente ambiental.

Ante la importancia de reducir el impacto del consumo de alcohol en los adolescentes, en este trabajo se explora la utilidad de un modelo teórico: la economía conductual, para analizar la preferencia por el consumo de alcohol, en función de las variables ambientales. Las investigaciones basadas en este modelo se han enfocado en responder tres preguntas: 1) ¿cómo varía el uso de la sustancia en función de las restricciones directas a su acceso (por ejemplo, mediante cambios en el precio -económico, monetario o conductual- o en la disponibilidad de la sustancia)? (DeGrandpre et al., 1993); 2) ¿cómo cambia el uso de la sustancia en función de las restricciones de otras actividades valiosas, como el trabajo, el ocio o las actividades sociales? (Vuchinich \& Tucker, 1988) y 3) ¿cómo cambia la distribución de la conducta cuando los consumidores eligen entre reforzadores que varían en cantidad y demora? (Bickel \& Marsch, 2001).

Esta última pregunta ha derivado en una importante línea de investigación sobre la elección intertemporal, donde se observa que los usuarios de sustancias sobrevaloran las recompensas inmediatas (por ejemplo, los efectos del alcohol) y restan importancia de una manera más pronunciada al valor de las recompensas demoradas (como en el estudio; Murphy et al., 2007). Debido al alcance del presente estudio (observacional), esta variable no se explora de manera directa; no obstante, permitirá explicar algunos resultados.

El hallazgo fundamental de los estudios, basados en la economía conductual, predice que el consumo es más probable en entornos con restricciones mínimas al acceso a la sustancia y elevadas limitaciones al acceso a reforzadores alternativos (Vuchinich \& Tucker, 1988). Este postulado se sustenta en resultados que muestran que: a) el consumo es altamente sensible a las alteraciones en su costo o precio; b) el uso de sustancias ocurre en mayor medida en contextos carentes de reforzadores alternativos libres de sustancias, y c) un mayor acceso a reforzadores alternativos resulta en una disminución del consumo (Correia et al., 2010; Higgins et al., 2004; Murphy \& Dennhardt, 2016; Vuchinich \& Heather, 2003).

En un entorno donde la disponibilidad del alcohol es constante, las restricciones a su acceso son bajas y la disponibilidad de actividades alternativas es limitada, la tarea prioritaria es el enriquecimiento ambiental mediante la disponibilidad de actividades alternativas. Es relevante resaltar que no todas las actividades libres de sustancias son incompatibles con la conducta de consumo (relación de sustitución) es decir, no todas tienen el potencial de disminuir el uso de sustancias (Hursh et al., 2005). La relación del consumo con algunas actividades también puede ser positiva (relación de complementariedad) incluso cuando no se realicen al mismo tiempo.

La identificación de las actividades que pueden competir con el uso de sustancias es una de las contribuciones más importantes para la prevención que pueden derivarse de este modelo. En ambientes naturales, las escalas de reforzamiento son el principal instrumento para identificar el tipo de relación que se establece entre las actividades libres de sustancias y la conducta de consumo (Correia et al., 1998). En estas escalas, el nivel de reforzamiento se mide utilizando dos dimensiones del reforzador: la frecuencia y el nivel subjetivo de placer. En este trabajo se retoman las experiencias obtenidas en estos estudios para elaborar un instrumento basado en las actividades que realizan los adolescentes mexicanos.

En el país, existe poca información acerca de cuáles son las actividades libres de sustancias incompatibles con el consumo de alcohol y de la influencia que tiene -en la conducta de consumo- la interacción entre el involucramiento en estas actividades y el acceso a la sustancia, en el entorno natural de los adolescentes. 
Por lo tanto, el objetivo del estudio fue evaluar el nivel de consumo de alcohol en un grupo de adolescentes mexicanos, en función del sexo, la edad, el acceso al consumo y el involucramiento en actividades libres de sustancias.

La meta del trabajo es brindar un sustento teórico acerca del efecto de las variables ambientales en la preferencia por el consumo de alcohol en los adolescentes. Los resultados pueden contribuir a incrementar el efecto de la prevención, al incorporar -en los programas que han mostrado ser efectivos- estrategias dirigidas a incrementar las restricciones al acceso al uso de alcohol y la disponibilidad de actividades incompatibles con esta conducta.

\section{MÉTODO}

\section{Diseño}

En este trabajo se describen los resultados de la tercera fase de un estudio más amplio (una descripción detallada de las fases previas se reporta en Alanís, 2019). En la primera fase (exploratoria), participaron 142 estudiantes. El objetivo fue identificar los indicadores para medir las principales variables de la economía conductual que explican el consumo de alcohol (acceso al consumo de alcohol y a las actividades libres de sustancias). Los datos fueron recolectados mediante dos técnicas (grupos focales y redes semánticas). Las unidades de información más frecuentes obtenidas mediante los grupos focales y las definidoras que conformaron el núcleo de la red en las redes semánticas, fueron utilizadas para elaborar los reactivos del instrumento de medición.

En la segunda etapa (piloteo y validación del instrumento) participaron 335 estudiantes. La confiabilidad de los reactivos se realizó mediante el Alfa de Cronbach. Los reactivos sobrevivientes se incluyeron en el análisis factorial (de componentes principales con rotación varimax), con el fin de identificar grupos homogéneos de variables (dimensiones).

En la tercera fase se aplicó la versión final del instrumento a 7,569 adolescentes. Se llevó a cabo un análisis de regresión lineal múltiple. Las variables predictoras que se ingresaron al modelo -además del sexo y la edadcorresponden a las dimensiones obtenidas mediante el análisis factorial (tipo de actividades y contextos de consumo); mientras que la variable dependiente fue el nivel de consumo de alcohol.

\section{Muestra}

El muestreo fue intencional; el criterio de inclusión fue ser estudiante de primero y segundo año de bachillerato en los planteles escolares donde se realizó el estudio; el de exclusión, no aceptar participar en la investigación. La muestra estuvo conformada por 7,569 estudiantes de bachillerato del Estado de México (ver Tabla 1). La edad promedio de los alumnos fue de $16.68(D E=0.68) 51 \%$ de los adolescentes fueron hombres y $49 \%$ mujeres.

\section{Escenario}

Las escuelas se ubicaron en zonas consideradas de riesgo para el consumo de sustancias, según los criterios establecidos en el Estudio Básico de Comunidad Objetivo ${ }^{1}$ (EBCO; Centros de Integración Juvenil, 2013). Las actividades se implementaron en los centros de cómputo de las instituciones educativas, debido a que el instrumento fue elaborado en formato digital.

\section{Procedimiento}

La actividad fue implementada por un grupo de docentes en coordinación con los responsables de los centros de cómputo de las instituciones, quienes recibieron capacitación sobre los aspectos técnicos para acceder al cuestionario y resolver los problemas relacionados. La versión digital del instrumento fue probada previamente por un grupo de estudiantes. Los docentes, una vez entrenados, brindaron las instrucciones a los estudiantes para realizar la tarea. Los participantes debían ingresar a una dirección electrónica y responder cada pregunta, seleccionando la opción de respuesta que mejor describiera sus experiencias. Al finalizar, sus respuestas eran enviadas a una base de datos. La tarea fue realizada en un promedio de 40 minutos.

\section{Mediciones}

La Prueba de Identificación de los Trastornos debido al Consumo de Alcohol (AUDIT, por sus siglas en inglés; Saunders et al., 1993) es un instrumento desarrollado por la Organización Mundial de la Salud para identificar el consumo de riesgo o perjudicial de alcohol, definido con base en el patrón de consumo y problemas asocia-

${ }^{1}$ EI EBCO define el nivel de riesgo a partir de cuatro grupos de factores: problemática familiar y escolar, deterioro de la calidad de vida, desintegración social e inseguridad pública. Las zonas de riesgo se clasifican en alto, medio y bajo. 
dos. Está integrado por 10 reactivos que representan tres dominios conceptuales: consumo de riesgo (patrón de consumo), consumo perjudicial (problemas asociados) y síntomas de dependencia. Las opciones de respuesta se presentan en una escala tipo Likert con una puntuación de cero a cuatro. El consumo de riesgo se identifica a partir de un punto de corte de ocho (AUDIT $\geq 8$ ). La validación en México con estudiantes de bachillerato se reporta en Gómez y colaboradores (2009). En esta investigación, en el análisis de regresión se utilizó el puntaje total de la escala como variable independiente, ya que brinda información más sensible acerca de los cambios en el nivel de consumo de alcohol.

Acceso al consumo de alcohol y actividades libres de sustancias, el instrumento de medición, elaborado para fines del estudio, está integrado por tres secciones:

I. Datos generales, dirigida a recopilar los datos sociodemográficos de los participantes.

II. Escala "actividades libres de sustancias", integrada por 18 reactivos, agrupados en 5 factores (tipos de actividades) que explican $51.0 \%$ de la varianza, con un Alfa de Cronbach de 0.78 ( $a=0.78)$. Los cinco grupos de actividades son: a) actividades culturales (realizar alguna actividad artística, asistir a actividades artísticas o culturales, leer y aprender una nueva actividad); b) uso de medios electrónicos (estar en redes sociales, navegar en Internet y escuchar música); c) actividades sociales (conocer personas, asistir a una fiesta sin alcohol, convivir con amigos y bailar); d) actividades académicas y prosociales (hacer tarea y estudiar, pensar acerca de tus planes futuros, ayudar en labores del hogar y colaborar como voluntario en actividades comunitarias), y e) actividades físicas y recreativas (hacer ejercicio, practicar un deporte individual e ir al cine). Para cada actividad se miden tres dimensiones del reforzador: a) frecuencia, por ejemplo, ¿con qué frecuencia ayudas en las labores del hogar?, las opciones van de nunca a tres o más veces por semana; b) nivel subjetivo de placer, por ejemplo, ¿qué tan agradable es o sería realizar la actividad?, las opciones van de nada agradable a muy agradable; y c) nivel de restricción, por ejemplo, ¿qué tan difícil es o sería realizar la actividad?, las opciones van de muy fácil a muy difícil.

III. Escala "acceso al consumo del alcohol", conformada por 12 reactivos, agrupados en dos factores (contextos de consumo) que explican $57.4 \%$ o de la varianza, con un Alfa de Cronbach de 0.90 ( $a=0.90)$. Los reactivos miden la frecuencia con la que los adolescentes tuvieron acceso al consumo en dos contextos: a) contexto social (beber alcohol en un antro o bar, en una reunión con amigos, en la calle, por invitación de un amigo, en el billar, en una fiesta y en la escuela), por ejemplo, ¿con qué frecuencia tuviste la oportunidad de ingerir alcohol en una fiesta?, y b) contexto familiar (consumo por invitación de un familiar, en una reunión familiar, en casa, al encontrar la sustancia en casa y por invitación de la pareja), donde se cuestionó ¿con qué frecuencia tuviste la oportunidad de consumir por invitación de un familiar? Las opciones de respuesta van de nunca a tres o más veces por semana.

\section{Análisis estadístico}

El análisis de los datos se realizó mediante el paquete estadístico SPSS versión 24 (SPSS Inc., 2016). Las diferencias entre hombres y mujeres en las variables sociodemográficas recolectadas se analizaron mediante la prueba Chi-cuadrado $\left(X^{2}\right)$. Se utilizó un modelo de regresión lineal múltiple, con el objetivo de evaluar de qué manera el sexo, la edad, el acceso al consumo y la preferencia, el placer y las restricciones asociadas con las actividades libres de sustancias (variables predictoras) se relacionan con el nivel del uso de alcohol (variable dependiente). El nivel de medición de todas las variables (con excepción del sexo) fue numérico. La variable independiente se midió utilizando el puntaje total del AUDIT. El criterio para incluir las variables en el análisis fue teórico, éstas fueron definidas a partir de los factores que conformaron el instrumento de medición, elaborado para fines de la investigación.

\section{Consideraciones éticas}

La participación de los estudiantes fue voluntaria; la información proporcionada, anónima, y el manejo de la información, estrictamente confidencial. Los participantes respondieron el instrumento en línea y no se solicitó ningún dato de identificación personal. Un grupo de docentes, previamente capacitados, solicitaron el consentimiento a los participantes de manera verbal. En el formato de consentimiento se describió el objetivo del estudio, la dinámica de la actividad, el carácter confidencial de la información, la libertad de los participantes de abandonar la tarea en el momento que lo desearan, así como los compromisos asumidos por la investigadora.

\section{Resultados}

La muestra estuvo integrada por 7,569 adolescentes de 15 a 18 años, con una edad promedio de $16.6(D=$ 0.68). $51 \%$ de los participantes fueron hombres y $49 \%$ mujeres; $47.3 \%$ estudiaba el primer año de bachillerato; $52.7 \%$, el segundo. Un poco más de la mitad, $53.4 \%$, eran alumnos del turno matutino y $46.6 \%$, del vespertino. $22.0 \%$ presentó un consumo de riesgo (AUDIT $\geq 8$ ): $24.9 \%$ de los hombres y $18.9 \%$ de las mujeres (ver Tabla 1 ). 
Se observaron diferencias significativas entre los sexos en la mayoría de las variables sociodemográficas recolectadas. Una mayor proporción de hombres reportó tener 18 años, más horas libres, mayor ingreso para sus gastos diarios y menor supervisión familiar. Estas variables han sido asociadas con un mayor riesgo de consumo (Rojas et al., 1999; World Health Organization, 2014).

En el modelo de regresión se introdujo como variable dependiente el nivel de consumo de alcohol; las variables predictoras fueron el sexo, la edad, el acceso al consumo en dos contextos (familiar y social), y tres dimensiones (frecuencia, nivel subjetivo de placer y nivel de restricciones) asociadas con los cinco grupos de actividades libres de sustancias (culturales, uso de medios electrónicos, sociales, académicas y prosociales, y físicas y recreativas; ver tabla 2). El modelo se ajustó $(F=202.423, p<0.01)$ y explicó $33.6 \%$ de la varianza $\left(R^{2}=0.336\right)$.

El sexo (ser hombre) y la edad fueron las variables asociadas con un mayor incremento en el nivel de consumo de alcohol en los adolescentes. El acceso a las bebidas en el contexto social-después del sexo y la edadfue la variable que más influyó en esta conducta. También el acceso en el contexto familiar también tuvo una influencia positiva en el nivel de consumo. Estos resultados resaltan la importancia de incrementar las restricciones al uso de alcohol, no sólo a partir de la regulación del mercado del alcohol y las restricciones en el entorno físico, sino también mediante cambios en las normas sociales y su percepción, así como en el establecimiento de reglas y el monitoreo dentro de las familias.

Las restricciones asociadas con las actividades libres de sustancias no tuvieron una relación significativa en el nivel consumo. La variable integra los reactivos que exploran el nivel de dificultad, percibida por los adolescentes para realizar los cinco tipos de actividades. El resultado contrasta con diversas observaciones, consistentes con la economía conductual, donde se describe una relación positiva entre el consumo de alcohol y las restricciones para tener acceso a las actividades valiosas libres de sustancias o a los obstáculos para involucrarse en éstas (Correia et al., 2002; Joyner et al., 2016).

Las actividades que mostraron una relación positiva con el nivel de consumo de alcohol fueron las sociales, las físicas y recreativas y el uso de medios electrónicos. Los hallazgos en torno al reforzamiento social son congruentes con la relevancia que tiene el contexto social entre los adolescentes y coindice con diversos trabajos que señalan una relación de complementariedad entre las actividades sociales y el uso de alcohol (Goldstein et al., 2014; Skidmore \& Murphy, 2010).
Con relación al uso de medios electrónicos, se ha observado que las personas que los utilizan de manera excesiva sobrevaloran la recompensa inmediata derivada de la actividad (Acuff et al., 2018), aunque también es posible que el alto nivel de valoración de estos medios (como Facebook o WhatsApp) se atribuya, en gran parte, a su recompensa social. La evidencia acerca de la relación entre el ejercicio y el deporte no es consistente, algunos estudios señalan que la relación positiva se observa principalmente en los deportes grupales y que la tendencia desaparece en los individuales (Wichstrøm \& Wichstrøm, 2009); sin embargo, también se ha descrito una relación positiva entre el consumo de alcohol y el ejercicio o la práctica de un deporte individual (Vancampfort et al., 2015).

Las actividades que mostraron una relación negativa con el nivel de consumo fueron las académicas y prosociales y las actividades culturales. El resultado sugiere que éstas tienen una relación de sustitución con la conducta de consumo; es decir, los adolescentes que las practican con más frecuencia y las consideran más gratificantes tienen menor nivel de consumo. En estudios previos, estas actividades (leer, pensar acerca de los planes futuros, actividades artísticas o académicas) han mostrado una relación negativa con el uso de sustancias (Correia et al., 2005; Hernández et al., 2016; Goldstein, et al., 2014).

En este trabajo no se midió directamente la relación entre el consumo de alcohol y la demora de la recompensa (descuento por la demora de la recompensa); sin embargo, una característica común entre las actividades identificadas como incompatibles con el consumo de alcohol es su reforzamiento demorado. En diversas investigaciones se ha observado que los adolescentes que consideran los resultados futuros al tomar decisiones actuales tienen menor probabilidad de usar sustancias (Amlung et al., 2012; Soltis, et al., 2017). La participación de los adolescentes en actividades orientadas hacia el futuro (el estudio, las actividades artísticas, prosociales), además de su potencial para prevenir y reducir el consumo de alcohol, puede favorecer su desarrollo personal, académico y social.

\section{DISCUSIÓN Y CONCLUSIONES}

En esta investigación se identificaron los contextos donde los adolescentes mexicanos tienen acceso al consumo de alcohol, así como las actividades libres de sustancias que, en el entorno natural de los adolescentes, tienen el potencial de competir con la conducta de consumo. Los resultados, consistentes con la perspectiva de la economía conductual, sugieren que el uso de alcohol en los adolescentes mexicanos se atribuye a las mínimas restricciones para beber, tanto en contextos sociales como 
Tabla 1

Descripción de la muestra, comparación entre hombres y mujeres

\begin{tabular}{|c|c|c|c|c|c|c|}
\hline \multirow{3}{*}{ Edad $^{\star *}$} & \multicolumn{2}{|c|}{$\begin{array}{c}\text { Hombres } \\
(n=3,863 ; 51 \%)\end{array}$} & \multicolumn{2}{|c|}{$\begin{array}{c}\text { Mujeres } \\
(n=3,706 ; 49 \%)\end{array}$} & \multicolumn{2}{|c|}{$\begin{array}{c}\text { Total } \\
(N=7,569 ; 100 \%)\end{array}$} \\
\hline & $\begin{array}{c}\bar{X} \\
16.72\end{array}$ & $\begin{array}{l}p \\
.70\end{array}$ & $\begin{array}{c}\bar{X} \\
16.65\end{array}$ & $\begin{array}{l}p \\
.65\end{array}$ & $\begin{array}{c}\bar{X} \\
16.68\end{array}$ & $\begin{array}{l}p \\
.68\end{array}$ \\
\hline & $f$ & $\%$ & $f$ & $\%$ & $f$ & $\%$ \\
\hline $\begin{array}{l}\text { Nivel de escolaridad } \\
\text { Primer año de bachillerato } \\
\text { Segundo año de bachillerato }\end{array}$ & $\begin{array}{l}1,795 \\
2,068\end{array}$ & $\begin{array}{l}46.5 \\
53.5\end{array}$ & $\begin{array}{l}1,788 \\
1,918\end{array}$ & $\begin{array}{l}48.3 \\
51.8\end{array}$ & $\begin{array}{l}3,583 \\
3,986\end{array}$ & $\begin{array}{l}47.3 \\
52.7\end{array}$ \\
\hline $\begin{array}{l}\text { Turno** } \\
\text { Matutino } \\
\text { Vespertino }\end{array}$ & $\begin{array}{l}1,981 \\
1,882\end{array}$ & $\begin{array}{l}51.3 \\
48.7\end{array}$ & $\begin{array}{l}2,063 \\
1,643\end{array}$ & $\begin{array}{l}57.7 \\
44.3\end{array}$ & $\begin{array}{l}4,044 \\
3,525\end{array}$ & $\begin{array}{l}53.4 \\
46.6\end{array}$ \\
\hline $\begin{array}{l}\text { Situación laboral } \\
\text { No trabaja } \\
\text { Trabaja }\end{array}$ & $\begin{array}{c}2,962 \\
901\end{array}$ & $\begin{array}{l}76.7 \\
23.3\end{array}$ & $\begin{array}{c}3,296 \\
409\end{array}$ & $\begin{array}{l}89.0 \\
11.0\end{array}$ & $\begin{array}{l}6,258 \\
1,310\end{array}$ & $\begin{array}{l}82.7 \\
17.3\end{array}$ \\
\hline $\begin{array}{l}\text { Ingreso monetario diario* } \\
\text { Menos de } \$ 30 \\
\text { De } \$ 30 \text { a } \$ 50 \\
\text { De } \$ 50 \text { a } \$ 100 \\
\text { Más de } \$ 100\end{array}$ & $\begin{array}{l}1,179 \\
1,751 \\
754 \\
179\end{array}$ & $\begin{array}{c}30.5 \\
45.3 \\
19.5 \\
4.6\end{array}$ & $\begin{array}{l}1,126 \\
1,832 \\
623 \\
125\end{array}$ & $\begin{array}{c}30.4 \\
49.4 \\
16.8 \\
3.4\end{array}$ & $\begin{array}{c}2,305 \\
3,583 \\
1,377 \\
304\end{array}$ & $\begin{array}{c}30.5 \\
47.3 \\
18.2 \\
4.0\end{array}$ \\
\hline $\begin{array}{l}\text { Horas libres diarias }^{\star *} \\
\text { De } 1 \text { a } 2 \\
\text { De } 3 \text { a } 4 \\
\text { De } 5 \text { a } 6 \\
\text { Más de } 6\end{array}$ & $\begin{array}{l}1,117 \\
1,782 \\
633 \\
331\end{array}$ & $\begin{array}{c}28.9 \\
46.1 \\
16.4 \\
8.6\end{array}$ & $\begin{array}{c}1,226 \\
1,667 \\
628 \\
185\end{array}$ & $\begin{array}{c}33.1 \\
45.0 \\
16.9 \\
5.0\end{array}$ & $\begin{array}{c}2,343 \\
3,449 \\
1,261 \\
516\end{array}$ & $\begin{array}{r}31.0 \\
45.6 \\
16.7 \\
6.8\end{array}$ \\
\hline $\begin{array}{l}\text { Horas libres fuera de casa** } \\
\text { Ninguna } \\
\text { De } 1 \text { a } 2 \\
\text { De } 3 \text { a } 4 \\
\text { Más de } 6\end{array}$ & $\begin{array}{l}1,008 \\
1,946 \\
585 \\
168\end{array}$ & $\begin{array}{c}26.1 \\
50.4 \\
15.1 \\
4.3\end{array}$ & $\begin{array}{c}1,411 \\
1,574 \\
418 \\
173\end{array}$ & $\begin{array}{c}38.1 \\
42.5 \\
11.3 \\
4.7\end{array}$ & $\begin{array}{c}2,419 \\
3,520 \\
1,003 \\
341\end{array}$ & $\begin{array}{c}32.0 \\
46.5 \\
13.3 \\
4.5\end{array}$ \\
\hline $\begin{array}{l}\text { Supervisión de los padres** } \\
\text { Siempre } \\
\text { Casi siempre } \\
\text { Casi nunca } \\
\text { Nunca }\end{array}$ & $\begin{array}{c}1,615 \\
1,837 \\
357 \\
54\end{array}$ & $\begin{array}{c}41.8 \\
47.6 \\
9.2 \\
1.4\end{array}$ & $\begin{array}{c}2,002 \\
1,347 \\
316 \\
41\end{array}$ & $\begin{array}{c}54.0 \\
36.3 \\
8.5 \\
1.1\end{array}$ & $\begin{array}{c}3,617 \\
3,184 \\
637 \\
95\end{array}$ & $\begin{array}{c}47.8 \\
42.1 \\
8.9 \\
1.3\end{array}$ \\
\hline $\begin{array}{l}\text { Con quién vive } \\
\text { Madre } \\
\text { Padre }^{\star *} \\
\text { Ambos padres }^{\star *}\end{array}$ & $\begin{array}{l}3,661 \\
3,177 \\
3,056\end{array}$ & $\begin{array}{l}94.8 \\
82.2 \\
79.1\end{array}$ & $\begin{array}{l}3,533 \\
2,871 \\
2,772\end{array}$ & $\begin{array}{l}95.3 \\
77.5 \\
74.8\end{array}$ & $\begin{array}{l}7,194 \\
6,048 \\
5,828\end{array}$ & $\begin{array}{l}95.0 \\
79.9 \\
77.0\end{array}$ \\
\hline $\begin{array}{l}\text { Problemas de consumo en la familia } \\
\text { Madre } \\
\text { Padre** } \\
\text { Hermanos }\end{array}$ & $\begin{array}{l}191 \\
797 \\
435\end{array}$ & $\begin{array}{c}4.9 \\
20.6 \\
11.3\end{array}$ & $\begin{array}{l}165 \\
853 \\
453\end{array}$ & $\begin{array}{c}4.5 \\
23.0 \\
12.2\end{array}$ & $\begin{array}{c}356 \\
1,650 \\
888\end{array}$ & $\begin{array}{c}4.7 \\
21.8 \\
11.7\end{array}$ \\
\hline $\begin{array}{l}\text { Prevalencia de consumo } \\
\text { Alguna vez en la vida* } \\
\text { Último año } \\
\text { Último mes } \text { me }^{* *} \\
\text { AUDIT } \geq 8^{* *}\end{array}$ & $\begin{array}{c}3,037 \\
2,465 \\
1,463 \\
961\end{array}$ & $\begin{array}{l}78.6 \\
63.8 \\
37.9 \\
24.9\end{array}$ & $\begin{array}{c}2,983 \\
2,355 \\
1,187 \\
701\end{array}$ & $\begin{array}{l}80.5 \\
63.5 \\
32.0 \\
18.9\end{array}$ & $\begin{array}{l}6,020 \\
4,820 \\
2,650 \\
1,662\end{array}$ & $\begin{array}{l}79.5 \\
63.7 \\
35.0 \\
22.0\end{array}$ \\
\hline
\end{tabular}

Nota: ${ }^{\star} p<.05 ;{ }^{* \star} p<.01$ 
Tabla 2

Modelo de regresión: consumo de alcohol, acceso al consumo y actividades libres de sustancias

\begin{tabular}{|c|c|c|c|c|c|c|}
\hline \multirow{2}{*}{ Variables } & & \multirow{2}{*}{$\begin{array}{l}\text { Coeficiente } \\
\text { de regresión }\end{array}$} & \multirow{2}{*}{$\begin{array}{c}\text { Coeficiente } \\
\text { estandarizado }\end{array}$} & \multirow{2}{*}{ Sig. } & \multicolumn{2}{|c|}{$\begin{array}{l}\text { Intervalo de confianza del } \\
\text { coeficiente de regresión }\end{array}$} \\
\hline & & & & & Limite inferior & Límite superior \\
\hline (Constante) & & -15.191 & & 0.000 & -18.52 & -11.85 \\
\hline Hombre & & 0.680 & 0.060 & 0.000 & 0.456 & 0.904 \\
\hline Edad & & 0.546 & 0.066 & 0.000 & 0.393 & 0.699 \\
\hline Contexto social & & 0.293 & 0.414 & 0.000 & 0.275 & 0.311 \\
\hline Contexto familiar & & 0.168 & 0.137 & 0.000 & 0.138 & 0.198 \\
\hline \multirow[t]{3}{*}{ Culturales } & Frecuencia & -0.011 & -0.009 & 0.519 & -0.046 & 0.023 \\
\hline & Placer & -0.073 & -0.034 & 0.040 & -0.143 & -0.003 \\
\hline & Restricciones & 0.007 & 0.003 & 0.852 & -0.067 & 0.081 \\
\hline Uso de medios & Frecuencia & 0.103 & 0.040 & 0.001 & 0.040 & 0.167 \\
\hline \multirow[t]{2}{*}{ electrónicos } & Placer & 0.068 & 0.017 & 0.219 & -0.040 & 0.175 \\
\hline & Restricciones & 0.006 & 0.001 & 0.914 & -0.095 & 0.106 \\
\hline \multirow[t]{3}{*}{ Sociales } & Frecuencia & 0.029 & 0.022 & 0.149 & -0.010 & 0.068 \\
\hline & Placer & 0.265 & 0.083 & 0.000 & 0.197 & 0.334 \\
\hline & Restricciones & -0.015 & -0.006 & 0.667 & -0.082 & 0.053 \\
\hline \multirow{3}{*}{$\begin{array}{l}\text { Académicas y } \\
\text { prosociales }\end{array}$} & Frecuencia & -0.065 & -0.036 & 0.003 & -0.108 & -0.023 \\
\hline & Placer & -0.090 & -0.034 & 0.014 & -0.162 & -0.018 \\
\hline & Restricciones & -0.017 & -0.006 & 0.639 & -0.086 & 0.053 \\
\hline \multirow[t]{3}{*}{ Físicas y recreativas } & Frecuencia & 0.060 & 0.037 & 0.006 & 0.017 & 0.104 \\
\hline & Placer & -0.052 & -0.017 & 0.241 & -0.140 & 0.035 \\
\hline & Restricciones & 0.075 & 0.025 & 0.084 & -0.011 & 0.160 \\
\hline
\end{tabular}

Nota: $F=202.423, g l=19, p<.01, R^{2}=0.336$

familiares, y al bajo reforzamiento (frecuencia y placer) derivado de las actividades incompatibles con el uso de alcohol. La incorporación de estos componentes en los programas de prevención que han mostrado ser efectivos, como los basados en el desarrollo de habilidades, puede contribuir a incrementar el impacto de las intervenciones.
Las estrategias dirigidas a incrementar las restricciones del consumo de alcohol se han enfocado en elevar el precio a nivel comercial y no consideran el costo de consumo en otros contextos, como el social y el familiar. Por ello, además de fortalecer las políticas públicas dirigidas a incrementar el costo monetario de la sustancia, un componente indispensable en los programas preventivos 
es la participación de la familia, la escuela y la comunidad. Estos sectores tienen un papel fundamental para incrementar las restricciones asociadas a la disponibilidad física de la sustancia y a las normas en torno a su uso.

Al mismo tiempo, es importante que las actividades incompatibles con el consumo de alcohol estén ampliamente disponibles en el entorno natural de los adolescentes y promover en los programas preventivos el involucramiento de los adolescentes en las mismas. La escuela y la comunidad son escenarios idóneos para incrementar el involucramiento de los adolescentes en estas actividades, por ejemplo, mediante clubes de lectura, actividades extracurriculares, clases de arte, exposiciones, tareas enfocadas en la orientación vocacional, etcétera. Es relevante incorporar un componente social en las actividades libres de sustancias para incrementar su nivel de reforzamiento, debido a que la mayoría de ellas se realizan de manera individual (estudio, lectura, actividades artísticas), mientras que las actividades que funcionan como complementos del consumo suelen ser sociales.

Finalmente, se señalan algunas limitaciones del estudio: a) el muestreo por conveniencia y la reducida variabilidad en la edad de los participantes son factores que limitan la generalización de los resultados a sectores más amplios, así como la observación de otras diferencias entre los grupos, y b) la medición de las variables no permitió identificar qué restricciones están asociadas con cada tipo de actividad ni las cualidades que influyen en su nivel de reforzamiento. En futuras investigaciones es esencial explorar alternativas para incrementar el reforzamiento derivado de las actividades incompatibles con el consumo de alcohol en el contexto familiar, escolar y comunitario.

\section{DECLARACIONES DE LOS AUTORES}

No existe ningún tipo de conflicto de interés.

\section{FUENTES DE FINANCIAMIENTO}

En este artículo se describen los resultados obtenidos en la última fase de un proyecto de tesis realizado por la autora principal durante sus estudios de doctorado en el Programa de maestría y doctorado de la Universidad Nacional Autónoma de México. Los estudios de doctorado fueron financiados por el Consejo Nacional de Ciencia y Tecnología.

\section{REFERENCIAS}

Acuff, S. F., Soltis, K. E., Dennhardt, A. A., Berlin, K. S., \& Murphy, J. G. (2018). Evaluating behavioral economic models of heavy drinking among college students. Alcoholism: Clinical \& Experimental Research, 42(7), 1304-1314. doi: 10.1111/acer.13774
Alanís, S. (2019). Uso de alcohol en adolescentes: acceso a la sustancia y disponibilidad de actividades alternativas [Tesis de doctorado publicada]. Universidad Nacional Autónoma de México.

Amlung, M. T., Acker, J., Stojek, M. K., Murphy, J. G., \& MacKillop, J. (2012). Is talk "cheap"? An initial investigation of the equivalence of alcohol purchase task performance for hypothetical and actual rewards. Alcoholism: Clinical and Experimental Research, 36(4), 716-724. doi: 10.1111/j.1530-0277.2011.01656.x

Babor, T., Caetano, R., Casswell, S., Edwards, G., Giesbrecht, N., Graham., K., Grube, J., Hill, L., Holder, H., Homel, R., Livingston, M., Österberg, E., Rehm, J., Room, R., \& Rosow, I. (2010). El alcohol: un producto de consumo no ordinario. Investigación y políticas públicas. (2.a ed.). Organización Panamericana de la Salud.

Bickel, W. K. \& Marsch, L. A. (2001). Toward a behavioral economic understanding of drug dependence: Delay discounting processes. Addiction, 96, 73-86. doi: 10.1046/j.1360-0443.2001.961736.x

Borges, G., Benjet, C., Medina-Mora, M. E., Orozco, R., \& Wang, P. S. (2008). Treatment of mental disorders for adolescents in Mexico City. Bulletin of the World Health Organization, 86(10), 757-764.

Borges, G., Orozco, R., Benjet, C., \& Medina-Mora, M. E. (2010). Suicidio y conductas suicidas en México: retrospectiva y situación actual. Salud Pública de México, 52(4), 292-304.

Centros de Integración Juvenil. (2013). Estudio Básico de Comunidad Objetivo, 2013. ClJ, Dirección de Investigación y Enseñanza, Subdirección de Investigación.

Correia, C. J., Benson, T. A., \& Carey, K. B. (2005). Decreased substance use following increases in alternative behaviors: a preliminary investigation. Addictive Behaviors, 30, 29-27. doi: 10.1016/j. addbeh.2004.04.006

Correia, C. J., Carey, K. B., \& Borsari, B. (2002). Measuring substance-free and substance-related reinforcement in the natural environment. Psychology of Addictive Behaviors, 16, 28-34.

Correia, C. J., Murphy, J. G., Irons, J. G., \& Vasi, A. E. (2010). The behavioral economics of substance use: research on the relationship between substance use and alternative reinforcers. Journal of Behavioral Health and Medicine, 3, 216-237. doi: 10.1016/j. ypmed.2016.04.022

Correia, C. J., Simons, J., Carey, K. B., \& Borsari, B. E. (1998). Predicting drug use: application of behavioral theories of choice. Addictive Behaviors, 23, 705-709. doi: 10.1016/S0306-4603(98)00027-6

Cuijpers, P. (2003). Three decades of drug prevention research. Drugs: education, prevention and policy, 10(1), 7-20. doi: 10.1080/0968763021000018900

DeGrandpre, R. J., Bickel, W. K., Hughes, J. R., Layng, M. P., \& Badger, G. (1993). Unit price as a useful metric in analyzing effects of reinforcer magnitude. Journal of the Experimental Analysis of Behavior, 60, 641-666. doi: 10.1901/jeab.1993.60-641

Foxcroft, D. R. \& Tsertsvadze, A. (2011). Universal school-based prevention programs for alcohol misuse in young people. Cochrane Database of Systematic Reviews, 5, CD009113. doi: 10.1002/14651858.CD009113.

Goldstein, A. L., Wall, A. M., Wekerle, C., \& Krank, M. (2014). The impact of perceived reinforcement from alcohol and in- 
volvement in leisure activities on adolescent alcohol use. Journal of Child \& Adolescent Substance Abuse, 22, 340-363. doi: 10.1080/1067828X.2012.735190

Gómez, E. L., Gómez, H. L., Morales, B., \& Pérez, M. (2009). Uso del AUDIT y el DAST-10 para la identificación de abuso de sustancias psicoactivas y alcohol en adolescentes. Revista Colombiana de Psicología, 18(1), 9- 17.

Guerrero, C. M., Muños, J. A., Sáenz, B., Pérez, R., \& Reynales, L. M. (2013). Impacto del consumo nocivo de alcohol en accidentes y enfermedades crónicas en México. Salud Pública de México, 55(2), 282-288.

Hernández, O., Espada, J. P., \& Guillén, A. (2016). Relación entre conducta prosocial, resolución de problemas y consumo de drogas en adolescentes. Anales de Psicología, 32(2), 609-616. doi: 10.6018/analesps.32.2.204941

Higgins, S. T., Heil, S. H., \& Lussier, J. P. (2004). Clinical implications of reinforcement as a determinant of substance use disorders. Annual Review of Psychology, 55, 431-461. doi: 10.1146/annurev. psych.55.090902.142033

Hursh, S. R., Galuska, C. M., Winger, G., \& Woods, J. H. (2005). The economics of drug abuse: a quantitative assessment of drug demand. Molecular Interventions, 5, 20-28. doi: 10.1124/mi.5.1.6

Joyner, K. J., Pickover, A. M., Soltis, K. E., Dennhardt, A. A., Martens, M. P., \& Murphy, J. G. (2016). Deficits in access to reward are associated with college student alcohol use disorder. Alcoholism: Clinical and Experimental Research, 4O(12), 2685-2691. doi: 10.1111/acer.13255

Medina-Mora, M. E., García, I., Cortina, D., Orozco, R., Robles, R., Vázquez, L. Real, T., \& Chisholm, D. (2010). Estudio de costo-efectividad de intervenciones para prevenir el abuso de alcohol en México. Salud Mental, 33(5), 373-378.

Murphy, J. G. \& Dennhardt, A. A. (2016) The behavioral economics of young adult substance abuse. Preventive Medicine 92, 24-30. doi: 10.1016/j.ypmed.2016.04.022

Murphy, J. G., Correia, C. J., \& Barnett, N. P. (2007). Behavioral economic approaches to reduce college student drinking. Addictive Behaviors, 32, 2573-2585. doi: 10.1016/j.addbeh.2007.05.015

Rojas, E, Fleiz, C., Medina-Mora, M. E., Moron, M. A., \& Domenech, M. (1999). Consumo de alcohol y drogas en estudiantes de Pachuca, Hidalgo. Salud Pública de México, 41, 297-308.

Saunders, J. B., Aasland, O. G., Babor, T. F., de la Fuente, J. R., \& Grant, M. (1993). Development of the Alcohol Use Disorders Identification Test (AUDIT): WHO collaborative project on early detec- tion of persons with harmful alcohol consumption. II. Addiction, 88, 791-804. doi: 10.1111/j.1360-0443.1993.tb02093.x

Skidmore, J. R. \& Murphy, J. G. (2010). Relations between heavy drinking, gender, and substance-free reinforcement. Experimental and Clinical Psychopharmacology, 18(2), 158-166. doi: 10.1037/ a0018513

Soltis, K. E., McDevitt-Murphy, M. E., \& Murphy, J. G. (2017). Alcohol demand, future orientation, and craving mediate the relation between depressive and stress symptoms and alcohol problems. Alcoholism: Clinical and Experimental Research, 41, 1191-1200. doi: 10.1111/acer.13395

Stockings, E., Hall, W. D., Lynskey, M., Morley, K. I., Reavley, N., Strang, J., Patton, G., \& Degenhardt, L. (2016). Prevention, early intervention, harm reduction, and treatment of substance use in young people. Lancet Psychiatry, 3(3) ,280-296. doi: 10.1016/ S2215-0366(16)00002-X

Vancampfort, D., De Hert, M., Stubbs, B., Soundy, A., De Herdt, A., Detraux, J., \& Probst, M. (2015). A systematic review of physical activity correlates in alcohol use disorders. Archives of Psychiatric Nursing 29(4), 196- 201. doi: 10.1016/j.apnu.2014.08.006

Villatoro, J. A., Fregoso., D., Bustos, M., Oliva, N., Mujica, A., Martín del Campo, R., Nanni, R., Medina-Mora, M. E. (2015). Encuesta Nacional de Consumo de Drogas en Estudiantes 2014. Reporte de alcohol. Instituto Nacional de Psiquiatría Ramón de la Fuente Muñiz, Comisión Nacional Contra las Adicciones, Secretaría de Salud, INPRFM.

Villatoro, J. A., Reséndiz, E., Mujica, A., Bretón M., Cañas, V., Soto, I., Fregoso, D., Fleiz, C., Medina, M. E., Gutiérrez, J., Franco, A., Romero, M. \& Mendoza, L. (2017). Encuesta Nacional de Consumo de Drogas, Alcohol y Tabaco 2016-2017: Reporte de alcohol. Instituto Nacional de Psiquiatría Ramón de la Fuente Muñiz, Instituto Nacional de Salud Pública, Comisión Nacional Contra las Adicciones, Secretaría de Salud.

Vuchinich, R. E, \& Tucker, J. A. (1988). Contributions from behavioral theories of choice to an analysis of alcohol abuse. Journal of $A b$ normal Psychology, 97, 181-195.

Vuchinich, R. E. \& Heather, N. (2003). Choice, behavioural economics, and addiction. Oxford, U.K: Pergamon.

Wichstrøm, T. \& Wichstrøm, L. (2009). Does sports participation during adolescence prevent later alcohol, tobacco and cannabis use? Addiction, 104, 138-149. doi: 10.1111/j.1360-0443.2008.02422.x

World Health Organization. (2014). Global status report on alcohol and health 2014. WHO Press: Switzerland. 